\title{
How Users Interact with Biodiversity Information Using TaxonTree
}

\author{
Bongshin Lee ${ }^{1,2}$, Cynthia Sims Parr ${ }^{1}$, Dana Campbell ${ }^{1}$, and Benjamin B. Bederson ${ }^{1,2}$ \\ ${ }^{1}$ Human-Computer Interaction Lab \\ ${ }^{2}$ Computer Science Department, \\ Univ. of Maryland, \\ College Park, MD 20742 \\ $+1-301-405-7445$
}

\{bongshin, csparr, bederson\}@cs.umd.edu and danac@wam.umd.edu

\begin{abstract}
Biodiversity databases have recently become widely available to the public and to other researchers. To retrieve information from these resources, users must understand the underlying data schemas even though they often are not content experts. Many other domains share this problem.

We developed an interface, TaxonTree, to visualize the taxonomic hierarchy of animal names. We applied integrated searching and browsing so that users need not have complete knowledge either of appropriate keywords or the organization of the data.

Our qualitative user study of TaxonTree in an undergraduate course is the first to describe usage patterns in the biodiversity domain. We found that tree-based interaction and visualization aided users' understanding of the data. Most users approached biodiversity data by browsing, using common, general knowledge rather than the scientific keyword expertise necessary to search using traditional interfaces. Users with different levels of interest in the domain had different interaction preferences.
\end{abstract}

\section{Categories and Subject Descriptors}

H.5.2 [Information Interfaces and Presentation]: User Interfaces - Graphical user interfaces (GUI), Interaction styles, Screen design, Evaluation/methodology; H.3.3 [Information Storage and retrieval]: Information Search and Retrieval Information filtering, Search process; J. 3 [Life and Medical Sciences]: Biology and genetics.

\section{General Terms}

Design, Experimentation, Human Factors.

\section{Keywords}

Biodiversity, Hierarchy/Tree Visualization, Information Retrieval, Searching, Browsing, Animation.

Permission to make digital or hard copies of all or part of this work for personal or classroom use is granted without fee provided that copies are not made or distributed for profit or commercial advantage and that copies bear this notice and the full citation on the first page. To copy otherwise, to republish, to post on servers or to redistribute to lists, requires prior specific permission and/or a fee.

AVI '04, May 25-28, 2004, Gallipoli (LE), Italy

(C) 2004 ACM 1-58113-867-9/04/0500..\$5.00

\section{INTRODUCTION}

\subsection{Biodiversity Information}

An understanding of the nature and magnitude of biological diversity is fundamental to most pressing environmental and conservation debates; the budding field of biodiversity informatics is poised to transform those debates [10, 21]. Biodiversity can be defined as "the variety of life" [28]. Typical questions in this field include: What kinds of organisms exist? What is their genetic diversity? How are these organisms distributed geographically? What processes are responsible for generating, maintaining, or changing this variety? How is this variety important to us and how do we affect it?

Biodiversity databases designed to answer these questions are as complex as molecular and medical biology resources. They contain organism-related information such as distribution, taxonomy, natural history, and conservation data. Perhaps the best developed are a number of taxonomic databases for researchers. The Species 2000 [1] initiative seeks a comprehensive index of the world's species. Museums provide online access to their specimen databases, often through gateways such as the Ocean Biogeographic Information System [4], and are currently developing tools for knowledge discovery and distributed querying (e.g. Mammal Networked Information System [3]).

The central challenge of biodiversity databases is that unlike molecular biology databases, complex biodiversity databases serve a broad audience often lacking content expertise. Wildlife departments and agricultural extension agencies maintain statespecific websites on local game, non-game, and pest species. These resources as well as commercial and private nonprofit natural history databases are aimed at concerned citizens and outdoor enthusiasts.

Whether they present primary data or encyclopedic summaries, these resources share a reliance on taxonomy, or the scientific naming of organisms. Biologists give organisms scientific names, usually Latin or Latin-like, that must follow certain rules to be considered official by the scientific community. Organisms thought to be close relatives are put into a group together, and related groups are likewise grouped, forming a nested hierarchy, which can be visualized as a tree. In a Linnaean classification, groupings receive a rank, such as Kingdom, Phylum, Class, Order, Family, Genus, and Species (in decreasing order of inclusiveness). Common names, on the other hand, are informal ways of referring to organisms. While they are not standardized (they differ 
according to language and dialect of the laypeople using them) common names can be very useful for non-experts.

\subsection{Biodiversity Information Retrieval}

Current interfaces to online biodiversity resources can be divided into three broad categories, none of which take advantage of current advances in HCI research such as dynamic queries [6], query previews [16], and visualization [13]. The simplest interfaces involve one or two free text query fields and either pulldown or radio button lists of categories to be searched. The current Animal Diversity Web interface is an example of such a simple structured query interface (Figure 1). This interface has the advantage of simplicity, but may not be used effectively by users who do not know currently recognized scientific or common names.

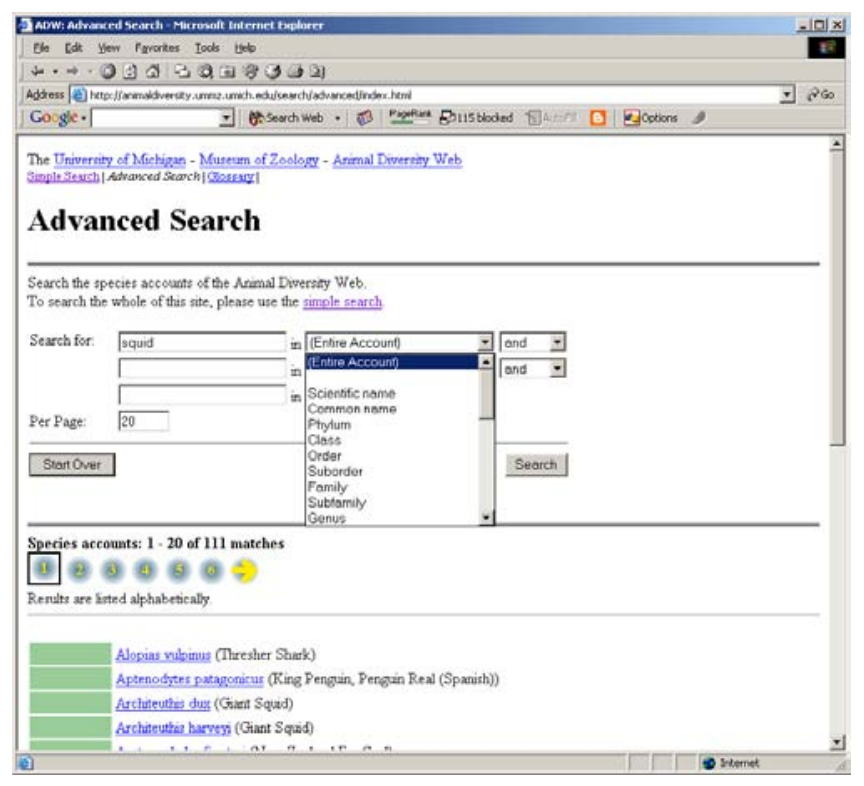

Figure 1. ADW Simple Structured Query Interface

Some interfaces rely heavily on controlled keyword choices and combine all possible structured queries on the same page. This kind of interface is typical of large conservation data resources, and is no doubt driven by the complexity of the different query types expected from users. While it reduces the need to know keywords for each category, a novice user or even a professional may be stymied by how much or how little of the page may be used for their search, and by the role of free text in the search.

The third and most complex kind combines the first two by providing different pages for different kinds of structured queries. This makes it possible to support many kinds of queries without including them all on the same page, but a user must determine which page has the query form of interest. It is possible to build a complex query across query types, but the user must build queries across pages.

These interfaces all have problems for end-users. First, they require that the users have significant knowledge about the underlying database, and the structure of the website search interface. Without such knowledge, users regularly get empty, or alternatively, huge search results - which are often meaningless. Second, they often provide search results in the form of a long list (Figure 1). Such lists lack biological context that could help users not only find specific information but understand the larger biodiversity patterns in the results. Our approach to solving these problems is to create an interface that couples browsing and searching of the taxonomic hierarchy using modern visualization techniques in order to help users build an understanding of the underlying data.

Below we review research on visualizing hierarchies, integrating searching and browsing, and other techniques that motivate our approach. We then describe the tool we designed, TaxonTree. Finally, we describe a user study of TaxonTree designed to characterize user behavior in the biodiversity domain.

\section{RELATED WORK}

\subsection{Visualizing Hierarchies}

Over the last 20 years there has been much research on how to effectively display and interact with hierarchies. One approach is node-link diagrams. Cone Trees, a 3D hierarchy visualization, makes the context easily visible with a focus and context display [25]. However, it has limits on scale and suffers from occlusions. Hyperbolic Browser solves the occlusion problems of Cone Trees by using hyperbolic space instead of 3D perspective [19]. WebTOC displays a hierarchical representation of the documents local to the site as a Table of Contents [23]. Users can expand and collapse the hierarchy. Links are listed with a bar that represents the volume of information available when following that link. PadPrints provides a dynamically built hierarchical history of visited web pages to aid web navigation [18]. Zooming enables users to control how much context is viewed. SpaceTree combines the node-link tree diagram with a zooming environment that dynamically lays out branches of the tree to best fit the available screen space [24]. Degree of Interest Trees compute a degree of interest value for each node in the hierarchy and display an overview of the complete hierarchy by only showing detail for nodes with high DOI values [12]. TreeJuxtaposer [22] also visualizes the entire hierarchy at once, but users select an area of any size to zoom into for more detail.

\subsection{Integrated Searching and Browsing}

A number of projects have integrated searching and browsing. SuperBook shows the number of occurrences of the search word to the left of section titles in a table of contents [15]. However, users still have to expand items manually to see how the words are distributed in the lower levels. AMIT (Animated Multiscale Interactive TreeViewer) integrates fisheye treebrowsing with search and filtering for web information access [29]. It displays only the paths to the matching nodes and the size of a node represent its relevance score. LifeLines searches can be performed on a patient record and highlights all parts of the record that match [7]. SpaceTree supports dynamic filters and searches showing results in the node link diagram [24]. Yee et al. applied integrated searching and browsing to user interfaces for large image collections within their web-based Flamenco system [30]. DateLens is a fisheye calendar that highlights the days containing appointments that match the search criteria [9]. In addition, its attribute-mapped scrollbar represents which days are highlighted outside of the current view.

\subsection{Animation}

Bederson and Boltman [8] found that animation improves users' ability to reconstruct the information space, and does not increase task performance time. In applications where the user navigates around fixed spatial data space, they recommend brief animated transitions. Others have made similar recommendations [11]. 


\subsection{SpaceTree}

Because SpaceTree [24] (Figure 2) combines hierarchy visualization, integrated searching and browsing, and animation, we chose to adapt SpaceTree to the biodiversity domain as a first step towards understanding that domain and its users. SpaceTree is a tree browser that enables users to explore large trees by adding a zooming environment that dynamically lays out branches to best fit the conventional node-link diagram to the available screen space.

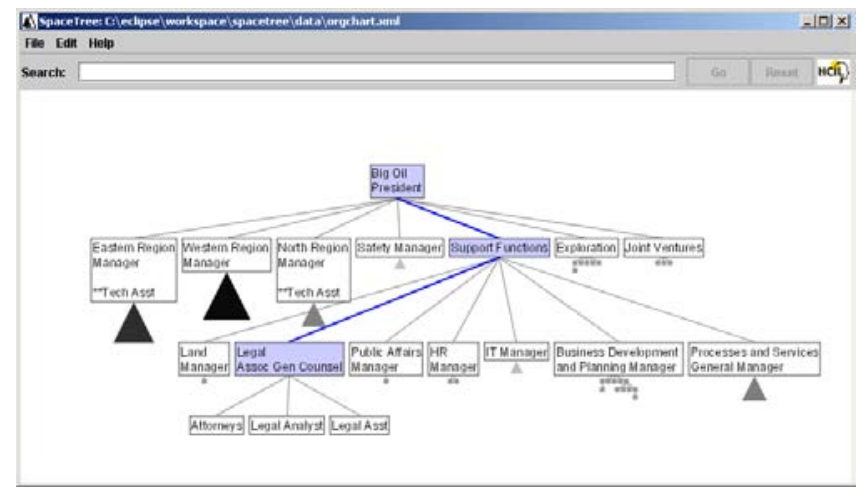

Figure 2. SpaceTree

Users can navigate the tree by clicking on nodes or by using the arrow keys. Based on user feedback that they didn't want to open the tree one level at a time when there was room to open more levels at once, SpaceTree maximizes the number of lower levels to be opened. When users change the focus, the tree is animated to its new layout, which makes full use of screen space, in three main steps: 1) trims the tree of the branches that would overlap the new branch to be opened; 2) moves the trimmed tree so that the new tree layout will center on the window, 3) expands the branch out of the new focus point. While animating, SpaceTree retains landmarks to help users maintain their orientation. It uses the current focus and the path up to the root as landmarks and highlights the ancestor path of the current focus. SpaceTree provides icons to preview the topology of branches that cannot be fully opened because of lack of space.

SpaceTree also supports filters and searches. As users type a string, SpaceTree highlights the relevant nodes within the tree. Users can see a filtered view of the tree, displaying only the paths to the matching nodes.

\section{TAXONTREE}

We developed ${ }^{1}$ TaxonTree (Figure 3 ) to visualize the Linnaean classification for taxonomic names in the Kingdom Animalia, by extending SpaceTree. TaxonTree allows users to browse and search a tree of about 200,000 animal names that we constructed by integrating data from a number of public and private sources $[2,5,17,20,26,27]$. TaxonTree, like SpaceTree, uses animated zooming and integrated searching and browsing. Search results are presented in the larger context of their classification tree to help users understand patterns in biodiversity information.

\footnotetext{
1 TaxonTree is available for download at www.cs.umd.edu/hcil/taxontree.
}

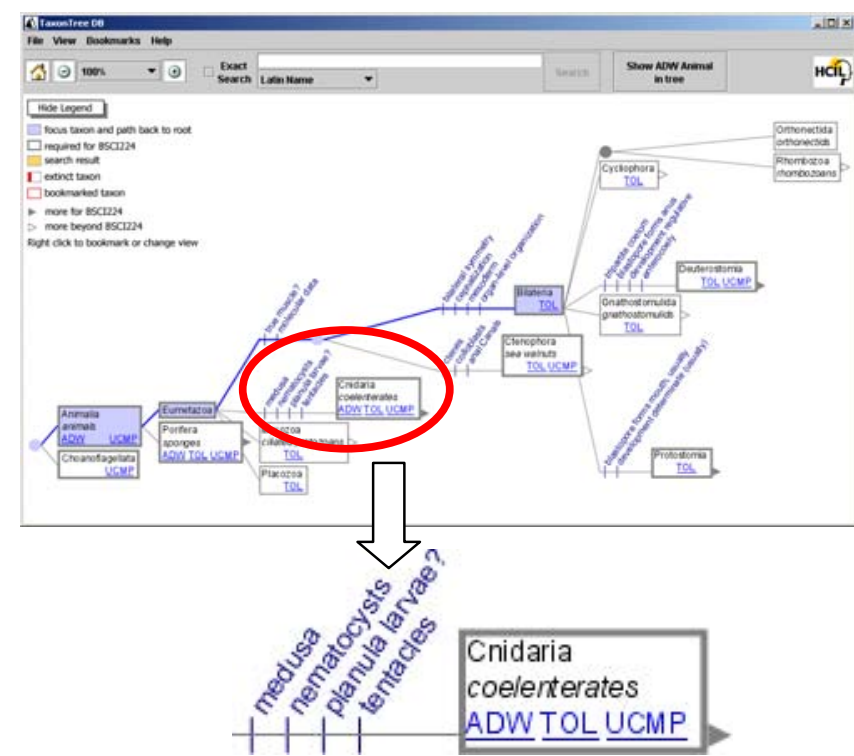

Figure 3. TaxonTree lays out branches of the classification tree to best fit the available screen space. Magnified nodes show synapomorphies (evolutionarily significant, diagnostic characteristics) and a small "more" triangle on the right side indicating that there are more nodes to be found by clicking on this node.

\subsection{Target Audience and Design Partners}

There have been no studies to describe usage patterns in the biodiversity information domain. We used methodologies adapted from collaborative design [14] to explore the domain and develop an interface for it in concert with its users.

Our target audience is students taking a second-year college course University of Maryland entitled Introduction to Animal Diversity. As biology majors, they are becoming familiar with the biological content but cannot be considered experts. To incorporate the extra detail that the course covered about known evolutionary relationships, we constructed a specialized tree of animal names that further resolved the Linnaean classification at higher levels [17, 20].

We were assisted by a team of five "design partners" who volunteered from the Animal Diversity course.

\subsection{Extensions}

Prior work [24] showed SpaceTree performed relatively well for both navigation and topology tasks for general tree visualization through a controlled study. However, since SpaceTree was developed and tested for general-purpose applications, we needed to extend it to accommodate domain specific requirements. While major functionalities are inherited from SpaceTree, TaxonTree expands on its capabilities in several ways.

\subsubsection{Domain-specific visualization}

We changed the visualization of TaxonTree to support biodiversity data. First, as you can see from the magnified node in Figure 3, we displayed common names with a different font style (italic) so that users can easily distinguish them from scientific names. Second, we provided links to external web pages on three different, publicly available websites (Animal Diversity Web, Tree of Life, University of California Museum of 
Paleontology). Thus, TaxonTree can be used as a portal to public biodiversity resources.

Some features were designed explicitly for the University of Maryland Animal Diversity course, such as visual distinction of required course material from non-required material, bookmarks of names for future reference, and display of biological ranks for each node as tool tips. We also added a way to see 'synapomorphies' (Figure 3) - attributes of nodes that show how that node is distinguished from its siblings. In TaxonTree, not only links but also nodes can have attributes.

\subsubsection{Search}

Users can search both scientific and common names in the classification. They can also search on synapomorphies and the full text of the Animal Diversity Web showing results within TaxonTree. TaxonTree presents search results in the biological context of their classification tree and highlights them (Figure 4). It helps users understand patterns in the results, for example animals with a common name including "dolphin" appear in two very different branches of the tree. Since the search result tree is often larger than screen, TaxonTree automatically zooms out to fit it in.

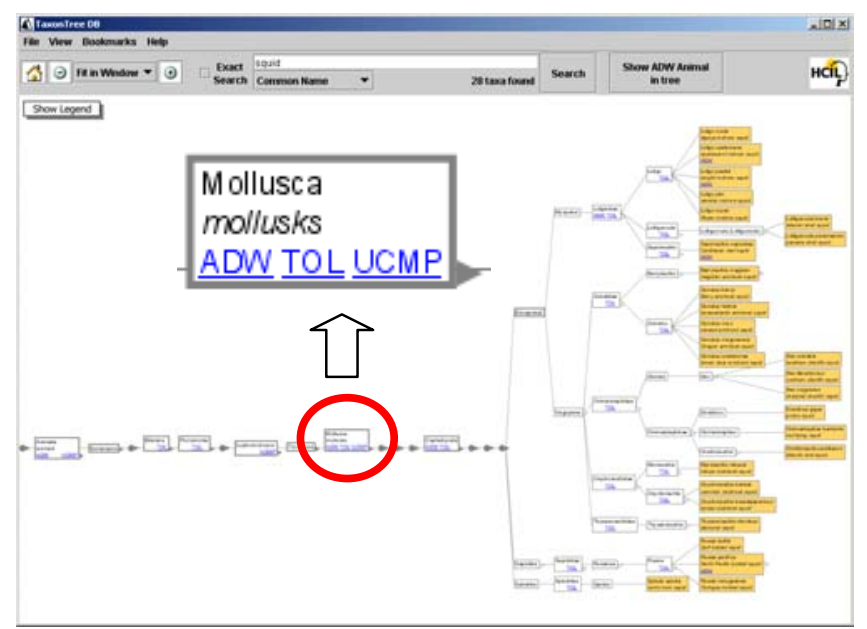

Figure 4. TaxonTree presents search results in the larger biological context of their classification tree. All squids are mollusks but there are several subgroups.

\subsubsection{Modified interaction}

As explained above, at the first step of animation when users change the focus, SpaceTree automatically closes the branches that would overlap a newly opened branch. This has two major advantages: 1) the screen is less cluttered; 2) the siblings of the focus node are always adjacent. However, we found that some of the more sophisticated 'auto-layout' features of SpaceTree were confusing and disorienting to our undergraduate design partners and project members with content experience and interest. We thus introduced an optional simpler navigation mode whereby users can open and close nodes manually.

\subsubsection{Scaling up}

To show the overview of the whole tree and to provide dynamic filters, SpaceTree requires the entire tree to be loaded into memory. Since this is impractical for large trees, we built TaxonTree to access the data from a database. In this way we are able to scale up to support interaction with very large trees and multiple attributes.

\section{USER STUDY}

In May 2003 we conducted a qualitative study with three main goals. First, we wanted to characterize how users of this domain think about biodiversity information in general. Are they more likely to look for information using scientific or common names? What taxonomic rank (species or higher) are they more likely to target? What kind of information are they most interested in? Second, we investigated the usability and interaction preferences with this particular software. Are users comfortable with integrated searching and browsing, and with animation and zooming? Finally, we examined how this kind of information retrieval interface can assist information understanding in this domain. Do students use the tree visualization to successfully complete tasks that require interpretation and understanding of the underlying data structure?

We chose a qualitative methodology because user behavior in this domain has never been studied. Also, the aim of TaxonTree is to foster content understanding so standard metrics of efficiency are unlikely to be appropriate. Insights gained from this study should guide both design and quantitative assessment of future tools.

\subsection{Participants}

We recruited 18 undergraduate volunteers ( 8 male: 10 female, 18 to 20 years old) from the Animal Diversity course at University of Maryland. None of them were part of the above described design partner team. Each participant was given ten dollars for his/her participation. We tested five pairs of users and eight single users for a total of 13 sessions, or user "teams." The study occurred at the end of the semester so participants were largely familiar with the biological content but could not be considered experts. The software had been demonstrated in lecture and distributed to all students on CD-ROM for personal use two weeks prior to the study. Users reported they had used the program for an average time of half an hour, and eight out of 18 had not used it at all.

\subsection{Procedure}

Each session lasted 30 to 45 minutes. Each user filled out a survey to determine their computer usage background and amount of time previously spent with TaxonTree. They were seated in front of a $2 \mathrm{GHz}$ Windows XP laptop with an ordinary mouse, a 1280x1024 pixel display and 512MB RAM, placed on a standard office desk. We videotaped the computer screen throughout the testing to capture both the actions and verbal comments of the users.

After briefly demonstrating TaxonTree features, we asked user teams to perform nine information retrieval tasks, described below. At the end of the tasks, we asked open-ended questions about what each user liked and found difficult about the software. To help characterize user needs, we asked what kind of information they generally would like about animals.

\subsection{Tasks}

Our goals were to learn how people approach information retrieval in this domain, and to learn if they could use our visualization tool to find and understand the information. Thus, we designed a range of tasks, described below, that included general tasks appropriate for any layperson as well as specific tasks related to our users' coursework.

Two general, open-ended tasks assessed user preferences for information targets and strategies to reach them. We asked them to use TaxonTree to find information about an animal of their choice. Depending on the strategy they took in task 1 (searching 
or browsing), we asked them in task 2 to choose another target animal and use the other strategy to find it.

The other seven tasks were more specific and had a limited number of correct answers. These tasks assessed a user's ability to use most of the features of the software, to further examine their preferences for information-seeking strategies, and to examine the role of the interface in understanding the information. These tasks were: 3 . Find an extinct taxon. 4. Count how many extinct taxa you might need to know about for the final exam. 5. Find and name the taxon whose members are all united with the synapomorphy "Lactation." 6 . What is the sister group to this group of lactating animals? 7. Now try searching on the common name, "dolphin." What do you notice about the results? 8. Find some victims or carriers of malaria. 9. What do you notice about these victims or carriers?

We recorded the information targets users chose and their initial strategy towards finding them (browsing or searching). We noted whether users completed a task and counted how many prompts we needed to give them so they could complete the task. Completing a task required a verbal indication that they had understood that the answer was onscreen. We noted what features of the tool elicited spontaneous positive reactions.

\section{RESULTS}

\subsection{Characterizing Users in This Domain}

We noticed during testing sessions that some users were clearly interested in the content. These users verbally expressed prior content knowledge as they worked on tasks, or asked questions indicating curiosity about information beyond the task. For example, a user asked "Why isn't there anything about mosquitoes?" when looking at result for a search about malaria. In contrast, some users never departed from the tasks at hand. Figure 5 illustrates how often users offered extra information indicating content interest. Guided by Figure 5, we defined high interest users as those who spontaneously offered extra content information during at least 4 of the 9 tasks; the others were labeled low interest. Consistent with this categorization, three users labeled low interest in this way had stated they lacked interest in the Animal Diversity course.

Low interest and high interest users reported similar hours of experience with the application and similar levels of comfort with computing. Six of the 8 males in the study were in low-interest user teams; two of the ten females were in low interest teams.

Users tended to be interested in looking for animals using common names and above the species level e.g. "frogs." (Order Anura). Specifically, when asked to choose any animal to find, users gave 20 out of 26 initial targets as common names rather than scientific names. Many targets were clearly above the species level (14 of 26 targets) while 4 were ambiguous and 8 were species level. Fewer than half of the search targets (12 of 26 targets) were required course content. Low and high interest user teams had similar search targets. When asked the kinds of information they were interested in, 5 of 13 teams mentioned way of life (food habits, behavior, ecology). Five of 13 teams noted that they enjoyed learning unique characteristics of animals interesting superlatives or what sets an animal apart from others. Four user teams mentioned that they wanted only the information necessary to pass their course. Two user teams mentioned an interest in evolutionary relationships. One user wanted information to distinguish dangerous from harmless animals.

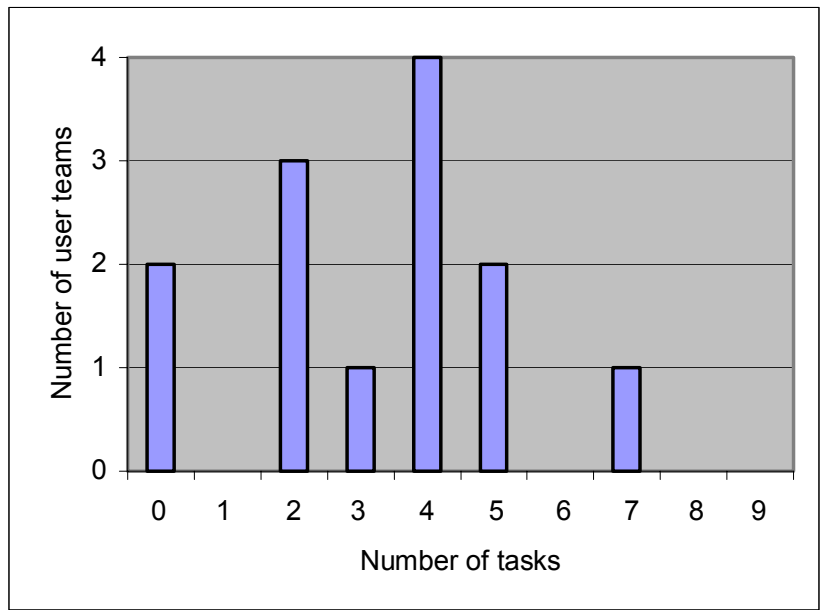

Figure 5. Distribution of 13 user teams based on the number of tasks in which they spontaneously offered additional biological information, indicating their interest in the domain

\subsection{Usability}

The interface seemed comfortable to users once they knew what features were available. Interaction with and interpretation of the nodes was apparently intuitive, because even users who had never used the program immediately began opening and closing nodes. Few users needed prompts explaining "more" triangles (shown in Figure 3), panning or zooming, node-clicking, or the ability to search. About $85 \%$ of the prompts we gave related instead to using our specific search categories and controlling the view options: how much of the tree was displayed (all nodes as they are opened, just the subset required for the course as they are opened, or all required nodes at once). Low interest users actually needed, on average, fewer prompts per session (4.3) to complete tasks compared to high interest users (7.4).

\subsection{Searching and Browsing}

Most users used both searching and browsing strategies together in at least one task. Only four user teams always used a single strategy within each task; three of these four teams were low interest users. Five of 13 teams browsed the tree before choosing a target or changed their target while browsing.

Most users preferred browsing the tree over searching. Only three of 13 teams used searching as an initial strategy - these were all high interest users who probably had better ideas of what search terms to use. Even after a successful search, 10 out of 11 subject teams returned to a strategy of browsing. When asked why, they told us it was more fun than searching ("I could have done a search for birds but this is more fun"). They also said that they wanted to refresh their memories, and that they didn't know exactly what to search on.

\subsection{Task Completion}

Users completed $92 \%$ of all tasks without prompts to interpret results shown onscreen.

Some tasks that asked for direct interpretation of the tree were very easy for the user teams. In task 5, 12 of 13 teams needed no prompts to correctly associate an attribute (the synapomorphy "lactation") with the name of the appropriate node. All but one team successfully completed task 6 , identifying a sister group from a search result by opening a nearby node. Only three teams needed a prompt. Task 9, what do you notice about victims and 
carriers of malaria, was readily answered. Eleven of 13 teams gave an immediate answer relating to the tree structure (such as, the search results were in related branches of the tree). Task 4 asked "Count how many extinct taxa you might need to know about for the final exam." All but one team immediately moved from displaying all overview nodes, including 16 color-coded as extinct, to task completion (counting all the nodes that were color coded as extinct).

However, some tasks were clearly harder than others. Task 7 asked users to draw inferences from a search on the common name "dolphin." We considered the task complete if the users gave at least one of two answers. First, there are many kinds of organisms whose common name includes the word "dolphin." Second, organisms with a common name including the word "dolphin" appear in more than one very different branches of the animal kingdom. The first inference was immediately drawn by all but one of the 13 user teams. Such an inference would be nearly impossible to make quickly using a typical list of search engine results. The second, however, requires the more sophisticated inference requiring an understanding of biological relationships. This inference was only mentioned by five of 13 user teams.

Task 8 asked users to conduct a search for carriers or victims of malaria. This task posed particular difficulty because of its sensitivity to both the search terms chosen and the category of search that was run. Three users were unable to complete the search without more than two prompts. These plus an additional two user teams failed to look at the web pages in the results to be sure that their search terms were in the appropriate context. However, 8 of 13 teams did check for relevance.

Tree visualization helped users complete tasks. Task 9, "What do you notice about these victims or carriers?" could be completed either by interpreting a tree visualization of search results or by applying prior knowledge to those search results. For example, a user response such as "all of these victims seem to live in forests" would be an example of prior knowledge, while "all of these victims are in the vertebrate part of the tree" indicates use of the tree to interpret the results. Although three or four user teams did use prior knowledge, only one set of users used it as their first answer to the question. All the others gave tree information for their first answer.

Domain interest seemed correlated with domain expertise as high interest users but not low interest users tended to effectively use their prior knowledge to help solve tasks.

\subsection{User Comments}

User responses to open ended questions are summarized in Table 1. Users said that TaxonTree was usable and had desirable content (synapomorphies, external web links, course information). Several mentioned explicitly that TaxonTree's visualization would be more useful to them than accessing the same information in their lecture notes or in the textbook.

Users had difficulty with unfamiliar features (search categories and view menu options). The other negative comments related to information quantity. Some users noted the difficulties inherent in displaying large amounts of information (font sizes, zooming problems). Some wanted more refined search results, while others felt that merely having so much information available to browse or search was daunting.
All but two user teams offered spontaneous positive comments while completing tasks. Visualizing search results in the tree structure elicited the most positive comments (6 user teams), along with the availability of web pages with more information (4). Four user teams also were excited about the ability to see an overview tree of the information necessary for their course.

Table 1. User comments to open-ended questions. Responses given by fewer than two user teams are not included.

\begin{tabular}{|lc|}
\hline What users liked & $\begin{array}{c}\text { \# of user } \\
\text { teams }\end{array}$ \\
Easy to learn and use & 9 \\
Synapomorphies & 9 \\
Ability to search different categories & 7 \\
How evolutionary history is presented & 6 \\
How tree is interactive & 4 \\
Links to external web sites. & 3 \\
Seeing which content required for their course. & 3 \\
What users found difficult & 3 \\
Search categories were hard to understand & 4 \\
Font too small, especially when zoomed out & 3 \\
Too much information, too many search results & 3 \\
Had problems zooming & 3 \\
Had problems understanding view menu & 3 \\
\hline
\end{tabular}

\section{DISCUSSION AND CONCLUSION}

While TaxonTree offers only incremental extensions to SpaceTree from the implementation point of view, it does point out how to make a specific domain-specific version of a generic visualization often requires a significant number of minor changes. More substantially, our qualitative user study of TaxonTree evaluated the software in a more natural domain-specific context than the prior SpaceTree study. Our test of TaxonTree used actual users of biodiversity information and tasks that require not only information retrieval but also information understanding.

Therefore our study provides two contributions: 1) the first description of how users in the biodiversity domain approach information retrieval; and 2) a richer demonstration of the value of interactive tree visualization with integrated searching and browsing.

\subsection{Characterizing Biodiversity Users}

As we originally argued, our data supports the idea that users of biodiversity data approach it without significant content expertise. Even interested biology majors primarily seek information using common names. They often look for general rather than specific names, and have some prior common knowledge about what they are looking for. Basic natural history is of interest, as well as particular characteristics distinguishing an animal from others. Though drawn from a course focusing on evolutionary relationships, only two user teams mentioned a special interest in them. This suggests a general lack of appreciation for the value of these relationships in understanding biodiversity. Our users expressed little concern over practical information such as which animals are dangerous, or how to care 
for pets. We recognize that our self-selected user sample is not representative of the public at large, but we have begun to characterize a subset of biodiversity information seekers: learners.

\subsection{Interactive Tree Visualization}

TaxonTree shows that interactive tree visualization can be applied to the biodiversity domain for a broad audience. A style of tree diagram (shown in Figure 6) that biology students are currently familiar with is very different from TaxonTree's style in the following ways: 1) it shows animal names only at leaves; 2) internal nodes are labeled with brackets outside of the tree; 3 ) every branch has a fixed angle. Despite these differences, users easily understood TaxonTree's tree structure. Our combination of interaction style and tree representation could therefore be useful in other domains, but a closer look at the trade-offs of the different representations is warranted.

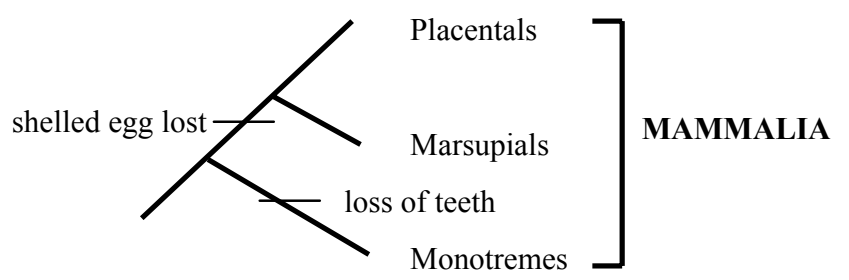

Figure 6. Example of tree diagram currently familiar to biology students

Users gave "it is fun" as one of several reasons they preferred to browse the tree rather than search it. Perhaps they enjoyed the animated interaction. If so, making interaction fun may be another benefit of animation, particularly in this often tedious domain.

It is beneficial to present search results in an interactive classification tree that shows biological context. Users easily interpreted search results, quickly using the tree structure to discover the quantity of biologically unique results. They often made more sophisticated inferences about relationships among the search results, which is nearly impossible by using a typical list of search results. They also carried out additional browsing, giving them a better sense of the search result by further browsing.

\subsection{Future Directions}

It is difficult to separate effects of content interest from content knowledge but we think this is a promising area for future research. In our study, high interest users often used prior knowledge to solve tasks. Was this because they had more knowledge (a consequence of their content interest) or were they more willing to use it?

After formal testing we asked some user teams to try the auto node closing feature. Their comments were consistent with the impressions we gained during our design phase: high interest user teams, like our design partners and our staff biologists, preferred manually closing and opening branches. Low interest users preferred auto-closing. We plan further tests to determine if this difference in interaction preference is really related to content expertise, and if so whether individuals transition from one style to the other as they become experts. Such differences in interaction preferences can be an issue for any application aimed at a broad audience.

\section{ACKNOWLEDGMENTS}

This work builds on our colleagues' work, and we especially thank Catherine Plaisant and Jesse Grosjean for their ongoing efforts on SpaceTree. We thank our design partners, L. Culler, V. Harrell, S. Little, S. Lutufsky, L.Noppinger, M. Pitts, A. Rung, and E. Thammasuvimol. Jeff Jensen, provided invaluable content advice and access to his undergraduate course. This work is supported by NSF \#0219492, by DARPA contract \#F30602-03-20018, and by IERI REC-0089283.

\section{REFERENCES}

[1] Species 2000 Federation (2000). http://www.sp2000.org.

[2] Integrated Taxonomic Information System (ITIS) (2003). http://www.itis.usda.gov.

[3] Mammal Networked Information System (MaNIS) (2003). http://elib.cs.berkeley.edu/manis.

[4] Ocean Biogeographic Information System (OBIS) (2003). http://www.iobis.org.

[5] University of Michigan Museum of Zoology (2003). http://www.ummz.umich.edu/birds.

[6] Ahlberg, C., and Shneiderman, B. (1994). Visual Information Seeking: Tight Coupling of Dynamic Query Filters With Starfield Displays. In Proceedings of Human Factors in Computing Systems (CHI 94) ACM Press, pp. 313-317.

[7] Alonso, D., Rose, A., Plaisant, C., and Norman, K. (1998). Viewing Personal History Records: A Comparison of Tabular Format and Graphical Presentation Using LifeLines. Behaviour and Information Technology, 17(5), pp. 249-262.

[8] Bederson, B. B., and Boltman, A. (1999). Does Animation Help Users Build Mental Maps of Spatial Information? In Proceedings of Information Visualization Symposium (InfoVis 99) IEEE, pp. 28-35.

[9] Bederson, B. B., Clamage, A., Czerwinski, M. P., and Robertson, G. G. DateLens: A Fisheye Calendar Interface for PDAs. ACM Transactions on Computer-Human Interaction, (in press).

[10] Bisby, F. A. (2000). The Quet Revolution: Biodiversity Informatics and the Internet. Science, 289, pp. 2309-2312.

[11] Card, S. K., Robertson, G. G., and Mackinlay, J. D. (1991). The Information Visualizer, an Information Workspace. In Proceedings of Human Factors in Computing Systems (CHI 91) ACM Press, pp. 181-188.

[12] Card, S., and Nation, D. (2002). Degree-of-Interest Trees: A Component of an Attention-Reactive User Interface. In Proceedings of Advanced Visual Interfaces (AVI 2002) ACM Press, pp. 231-245.

[13] Card, S. K., Mackinlay, J. D., and Shneiderman, B. (1999). Information Visualization. S. K. Card, J. D. Mackinlay, and B. Shneiderman (eds.), Readings in Information Visualization: Using Vision to Think (pp. 1-34). California: Morgan Kaufmann.

[14] Druin, A. (1999). Cooperative Inquiry: Developing New Technologies for Children With Children. In Proceedings of Human Factors in Computing Systems (CHI 99) ACM Press, pp. 223-230.

[15] Egan, D. E., Remde, J. R., Gomez, J. M., Landauer, T. K., Eberhardt, J., and Lochbaum, C. C. (1989). Formative 
Design-Evaluation of SuperBook. ACM Transactions on Information Systems, 7(1), pp. 30-57.

[16] Greene, S., Tanin, E., Plaisant, C., Shneiderman, B., Olsen, L., Major, G., and Johns, S. (1997). The End of Zero-Hit Queries: Query Previews for NASA's Global Change Master Directory. International Journal Digital Libraries, 2(2+3), pp. 79-90.

[17] Hickman, C. P., Roberts, L. S., and Larson, A. (2003). Animal Diversity, 3rd Edition. McGraw-Hill.

[18] Hightower, R. R., Ring, L., Helfman, J., Bederson, B. B., and Hollan, J. D. (1998). Graphical Multiscale Web Histories: A Study of PadPrints. In Proceedings of ACM Conference on Hypertext (Hypertext 98) ACM Press, pp. 58-65.

[19] Lamping, J., Rao, R., and Pirolli, P. (1995). A Focus+Context Technique Based on Hyperbolic Geometry for Visualizing Large Hierarchies. In Proceedings of Human Factors in Computing Systems (CHI 95) ACM Press, pp. 401-408.

[20] Maddison, D. R. (ed.). Tree of Life (2003). http://www.tolweb.org.

[21] Maier, D. E., Landis, J., Cushing, A., Frondorf, A., Silberschatz, M., Frame, J. L., and Schnase. (2000). Research Directions in Biodiversity and Ecosystem Informatics. NASA Goddard Space Flight Center, Maryland.

[22] Munzner, T., Guimbretière, F., Tasiran, S., Zhang, L., and Zhou, Y. (2003). TreeJuxtaposer: Scalable Tree Comparison Using Focus+Context With Guaranteed Visibility. In Proceedings of the 30th annual conference on Computer graphics and interactive techniques (SIGGRAPH 2003) ACM Press, pp. 453-462.

[23] Nation, D. A., Plaisant, C., Marchionini, G., and Komlodi, A. (1997). Visualizing Websites Using a Hierarchical Table of Contents Browser: WebTOC. (Third Conference on Human factors and the Web)

[24] Plaisant, C., Grosjean, J., and Bederson, B. B. (2002). SpaceTree: Supporting Exploration in Large Node Link Tree, Design Evolution and Empirical Evaluation. In Proceedings of Information Visualization Symposium (InfoVis 2002) IEEE, pp. 57-64.

[25] Robertson, G. G., Mackinlay, J. D., and Card, S. K. (1991). Cone Trees: Animated 3D Visualizations of Hierarchical Information. In Proceedings of Human Factors in Computing Systems (CHI 91) ACM Press, pp. 189-194.

[26] Uetz, P. (ed.). European Molecular Biology Laboratory Reptile Database (2003). http://www.reptile-database.org.

[27] Wilson, D. E., and Reeder, D. M. (eds.). Mammal Species of the World (1993). http://www.nmnh.si.edu/msw.

[28] Wilson, E. O. (1992). The Diversity of Life. New York: Norton.

[29] Wittenburg, K., and Sigman, E. (1997). Visual Focusing and Transition Techniques in a Treeviewer for Web Information Access. In Proceedings of Visual Languages (VL 97) IEEE, pp. 20-27.

[30] Yee, K.-P., Swearingen, K., Li, K., and Hearst, M. (2003). Faceted Metadata for Image Search and Browsing. In Proceedings of Human Factors in Computing Systems (CHI 2003) ACM Press, pp. 401-408. 